\title{
Experimental Tests of
}

\section{Charge Symmetry Breaking in Hypernuclei}

\author{
Patrick Achenbach* \\ Institut für Kernphysik, Johannes Gutenberg-Universität, Mainz, Germany \\ E-mail: achenbach@uni-mainz.de
}

\begin{abstract}
A pedagogical discussion of the charge symmetry breaking effect in light hypernuclei is provided. The topic is addressed because recent hypernuclear decay measurements at the electron accelerator MAMI in Germany and at the J-PARC hadron hall in Japan combined with older nuclear emulsion data independently confirmed the existence of $\Lambda$ binding energy differences between the lightest mirror pair of hypernuclei in the ground and in the excited states. In this particular pair $\left({ }_{\Lambda}^{4} \mathrm{He},{ }_{\Lambda}^{4} \mathrm{H}\right)$ charge symmetry breaking appears to be considerably stronger than in any other nuclei or heavier hypernuclei. This article is not intended to be a comprehensive overview of these experiments and their results which already have been published in the last year.
\end{abstract}

54th International Winter Meeting on Nuclear Physics

25-29 January 2016

Bormio, Italy

${ }^{*}$ Speaker. 


\section{Introduction}

Charge symmetry of the strong interaction is manifest in the symmetry of nuclear properties under the exchange of protons and neutrons. The near equality of the mass of these two particles on the level of $\Delta M / M=1.4 \times 10^{-3}$ suggests an underlying symmetry. However, Coulomb effects break this symmetry since a charge $Z e$ is present within the nuclear volume. In experiments with neutrons and protons the charge symmetry of the strong interaction can only be probed if the observations are corrected for Coulomb effects or if they can be neglected. The saturation of the binding energy in nuclei is independent of mass number $A$ and charge number $Z$ and represents charge independence. Historically, its observation lead to the first successful model to describe nuclear properties, the liquid drop model, that incorporated charge independence. In the absence of Coulomb interactions, a charge-symmetric and charge-independent nuclear force would result in identical binding energies and level schemes of mirror nuclei, that have their proton and neutron numbers exchanged. The symmetry in the interaction was first recognized through the analysis of the $N N$ scattering lengths for the $p p, n n$, and $n p$ systems, that would be identical in the spin-singlet channel without Coulomb interactions. The measurements suggest that the interaction in the $n n$ channel is only about $1 \%$ more attractive than in the $p p$ channel and $n p$ is approximately $2 \%$ more attractive than $p p$ and $n n$ [1].

The charge symmetry principle is a particular case of the more fundamental principle of isospin invariance in strong interactions, including not only the two nucleon states with isospin $z$-component $I_{z}= \pm 1 / 2$ for the proton and the neutron, but all mesons and baryons, among them the isospin zero singlet of $\Lambda$ hyperons and the $\Delta M_{\Lambda \Sigma} \sim 80 \mathrm{MeV} / c^{2}$ more massive isospin triplet of $\Sigma$ hyperons. In the isospin formalism, the charge symmetry operation corresponds to a rotation in isospin of $180^{\circ}$ about an axis perpendicular to the $z$ axis. For states with equal neutron and proton number it follows that $I_{z}=0$. The Coulomb interaction primarily adds to the nuclear energy a term depending on $I_{z}$. However, also isospin violating effects could be contained, e.g. the tendency of the Coulomb interaction to push the protons towards the nuclear surface. In the semi-empirical liquid drop model for the nuclear binding energy the only $I_{z}$ dependent term is the Coulomb energy. Thus, the binding energy difference between isobaric analog states which have the same $A$ and $I$ but different $I_{z}$ is given in this model only by the Coulomb energy. For the lightest nuclei, this leads to only small energy splittings between the isobaric multiplets.

Charge symmetry also predicts that the $\Lambda p$ and $\Lambda n$ interactions and consequently their contributions to the binding energies of mirror hypernuclei would be identical. The binding energy, $B_{\Lambda}$ of a $\Lambda$ hyperon in a hypernucleus, correctly referred to as the separation energy $S_{\Lambda}$, represents the difference between the hypernuclear mass equivalent in the ground state and the mass equivalents of the separated hyperon and core. It is given by

$$
-B_{\Lambda}=M\left({ }_{\Lambda}^{A} Z\right) c^{2}-M\left({ }^{A-1} Z\right) c^{2}-M_{\Lambda} c^{2}
$$

and can be determined by measuring the kinetic energies of all disintegration products when the hypernucleus decays by one of the possible modes

$$
\begin{aligned}
& { }_{\Lambda}^{A} Z \rightarrow{ }^{A} Z+\left(M_{\Lambda}-M_{n}\right) c^{2}-B_{\Lambda} \\
& { }_{\Lambda}^{A} Z \rightarrow{ }^{A}(Z+1)+\left(M_{\Lambda}-M_{n}-M_{\pi-}\right) c^{2}-B_{\Lambda} .
\end{aligned}
$$




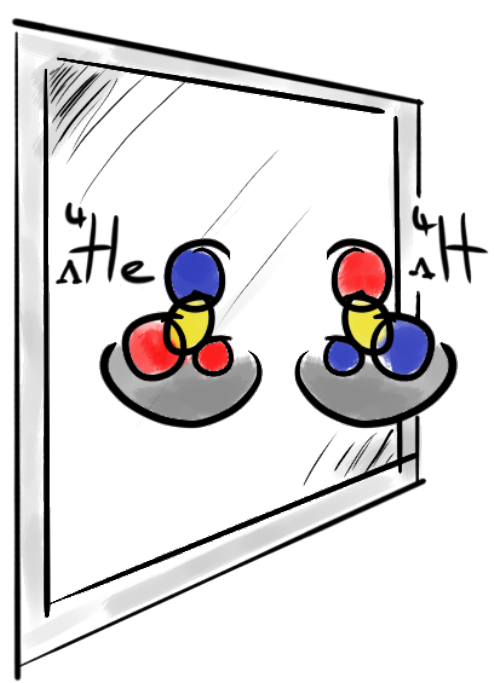

Figure 1: The concept of charge symmetry in nuclear forces extended to nuclei with strangeness leads to charge-conjugate hypernuclei like ${ }_{\Lambda}^{4} \mathrm{H}$ and ${ }_{\Lambda}^{4} \mathrm{He}$ that would be structurally identical in their ground and excited states. The illustrated mirror transforms the $z$-component of the isospin quantum number of the mirror hypernuclei. Note, that the $\Lambda$ hyperon is an uncharged isospin zero singlet and both $A=4$ hypernuclei have isospin $I=1 / 2$ with $I_{z}=-1 / 2$ for ${ }_{\Lambda}^{4} \mathrm{H}$ and $I_{z}=+1 / 2$ for ${ }_{\Lambda}^{4} \mathrm{He}$.

There are several light hypernuclei forming isobaric doublets such as $\left({ }_{\Lambda}^{4} \mathrm{H},{ }_{\Lambda}^{4} \mathrm{He}\right)$ and $\left({ }_{\Lambda}^{8} \mathrm{Li},{ }_{\Lambda}^{8} \mathrm{Be}\right)$, while ${ }_{\Lambda}^{7} \mathrm{He}$ and ${ }_{\Lambda}^{7} \mathrm{Be}$ belong to an isobaric triplet with ${ }_{\Lambda}^{7} \mathrm{Li}$. Fig. 1 illustrates the example of the lightest pair of mirror hypernuclei with exchanged z-component of the isospin quantum number. The experimental values of $B_{\Lambda}$ for the mirror pairs and in the isobaric analog states are found to be approximately the same and give some evidence for charge symmetry in the interaction of the $\Lambda$ hyperon with nucleons [2].

\section{Charge symmetry breaking}

Observations of charge symmetry breaking (CSB) in nuclei can be linked to the underlying forces and the emergence of nuclear structure properties. The main source of CSB comes from Coulomb interactions. In the semi-empirical liquid drop model approach the Coulomb energy of a uniform nucleus with charge radius $R$ assuming point-like nucleons can be calculated to be

$$
E_{C}^{\text {point }}(Z)=\frac{3}{5} \cdot \frac{1}{4 \pi \varepsilon_{0}} \frac{Z^{2} e^{2}}{R}, \quad \text { or } \quad E_{C}(Z)=\frac{3}{5} \cdot \frac{1}{4 \pi \varepsilon_{0}} \frac{Z(Z-1) e^{2}}{R}
$$

when accounting for a smeared charge density of the protons. The Coulomb energy difference $\Delta E_{C}$ of mirror pairs with charge numbers $Z$ and $Z-1$ then becomes

$$
\Delta E_{C}=E_{C}(Z-1)-E_{C}(Z)=\frac{3}{5} \cdot \frac{1}{4 \pi \varepsilon_{0}} \frac{2(Z-1) e^{2}}{R}
$$

from which already an order-of-magnitude correct estimate of the Coulomb contribution can derived. As an example, the static Coulomb energy difference in the $A=3$ isodoublet $\left({ }^{3} \mathrm{H},{ }^{3} \mathrm{He}\right)$ can 
be estimated to be $\left|\Delta E_{C}\right|<1 \mathrm{MeV}$ using an isoscalar charge radius of $R^{3} \sim 1.9 \mathrm{fm}$, consistent with estimates of the Coulomb effect from the binding energy difference. It is clear that in light nuclei the Coulomb energy cannot be described correctly by a simple expression such as Eq. 2.1 partly because the diffuseness in the charge distribution is comparable to the nuclear radius and partly because the neutron-proton exchange term becomes more important and depends on details of the nuclear configuration [1].

A very fundamental CSB is the small difference between the masses of $u$ - and $d$-quarks. However, the nuclear interaction at large distances is more conveniently described in terms of the exchange of mesons. A special role is played by the one-pion $(I=1)$ exchange due to the smallness of the pion mass of $M_{\pi} \approx 140 \mathrm{MeV} / c^{2}$ and the corresponding long Compton wavelength $\lambda_{c}=\hbar / M c \simeq 1.4 \mathrm{fm}$. In meson exchange models CSB is generated by the difference between the masses of the neutral and charged mesons. The difference of the $n n$ and $p p$ scattering lengths as well as the remaining binding energy difference in the $A=3$ mirror nuclei after correcting for Coulomb effects is reproduced quantitatively by taking the mixing of $\rho^{0} \omega$ into account [3].
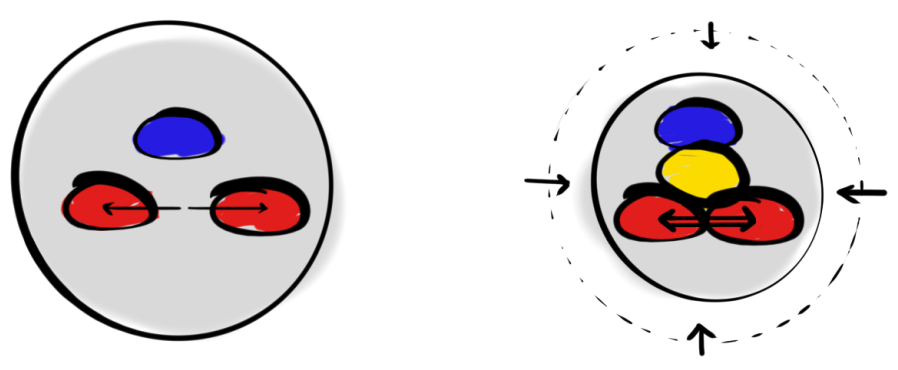

Figure 2: The shrinking of the core of a hypernucleus due to the presence of the additional bound hyperon leads to a difference in Coulomb energy contributing to charge symmetry breaking in mirror pairs. It is expected that the $\Lambda$ binding energy is smaller for the hypernucleus with larger charge number in comparison to its mirror partner.

The $\Lambda N$ interaction is weaker than the $N N$ interaction, which can partly be attributed to the fact that the one-pion exchange between the isospin zero singlet $\Lambda$ and a nucleon is forbidden in isospinconserving interactions except for electromagnetic violations via $\Lambda-\Sigma^{0}$ mixing [2,4]. Under the operation of charge exchange, i.e. exchange of $z$-component of the isospin, the $\Lambda$ state remains unchanged, whereas the $\Sigma^{0}$ state changes sign. If an appreciable mixing between the isospin-pure $\Sigma^{0}$ and $\Lambda$ states is realized in nature, the physical eigenstate $\Lambda_{\text {phys }}$ is no longer isospin-pure and is not an eigenstate of the charge operator. The effect of admixture therefore breaks charge symmetry and leads to a binding energy difference in mirror hypernuclei as first pointed out by Dalitz and von Hippel [5]. The interaction also involves the coupled $\Lambda N$ and $\Sigma N$ channels, in which $I=1$ meson exchanges such as $\pi$ or $\rho$ contribute as well as the two-pion exchange three-body interaction [6]. The coupling is much more important as compared to $N N$ interactions because of the missing longrange one-pion exchange in $\Lambda N$ and the smaller mass difference with respect to the $N \Delta$ transition energy of $E_{N \Delta} \sim 300 \mathrm{MeV}$.

In hypernuclei the presence of the $\Lambda$ hyperon compresses the core nucleus leading to an increase in Coulomb energy for the hypernucleus with larger charge number as compared to its 

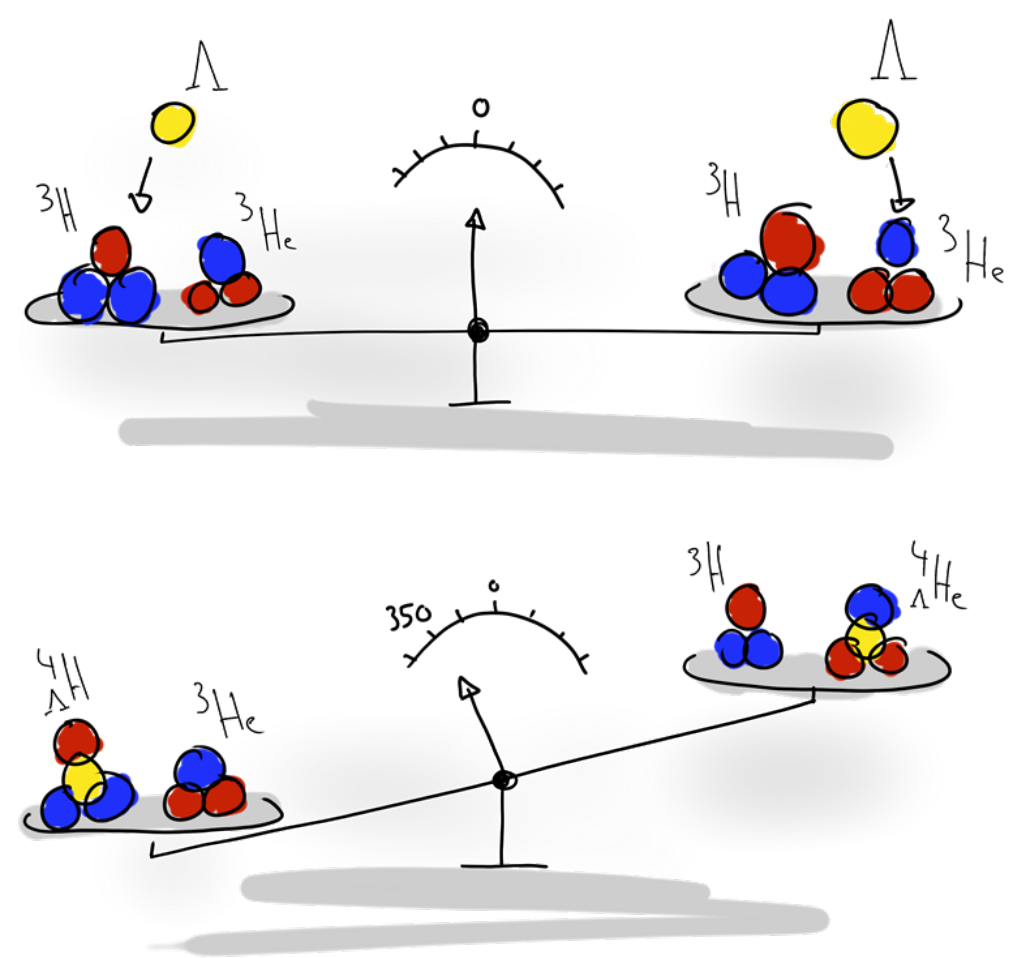

Figure 3: Top: charge symmetry in the nuclear force predicts the binding energies of the $\Lambda$ hyperon in the mirror pair of $A=4$ hypernuclei to be identical which would lead to equal masses for the sum of ${ }^{3} \mathrm{H}$ and ${ }^{3} \mathrm{He}$ nuclei plus one bound $\Lambda$ hyperon. Bottom: experimental evidence for charge symmetry breaking in $A=4$ hypernuclei comes from nuclear emulsion data where an exceptionally large ground-state binding energy difference of $\Delta B_{\Lambda}^{4}\left(0_{\mathrm{g} . s .}^{+}\right) \approx+0.35 \mathrm{MeV}$ was observed.

mirror partner. The effect is sketched in Fig. 2. The corresponding change in binding energy is expected to be negative for hypernuclear mirror pairs. In the system of $A=4$ hypernuclei nuclear emulsion measurements found an exceptionally large and positive difference of $\Delta B_{\Lambda}^{4}\left(0_{\text {g.s. }}^{+}\right) \approx$ $+0.35 \pm 0.05 \mathrm{MeV}$ between the ground state binding energies of the ${ }_{\Lambda}^{4} \mathrm{H}$ hypernucleus and the charge-conjugate ${ }_{\Lambda}^{4} \mathrm{He}$ hypernucleus [7]. Fig. 3 illustrates the mass differences in this system. This ground state difference is considerably larger than the $\Delta B^{3} \approx 0.07 \mathrm{MeV}$ assigned to and consistently calculated CSB splitting in the mirror core nuclei. Furthermore, the shrinking effect is increasing the difference attributed to the strong interaction and the CSB of the excited states differs significantly from the ground state. To obtain by calculation the correct binding energies from fundamental interactions especially for this lightest pair of mirror hypernuclei is not trivial and was resisting a consistent reproduction by theory until recently [8].

\section{From nuclear emulsion and modern experimental techniques}

Early studies of hypernuclei were performed mainly using the nuclear emulsion technique 
from which the ground state binding energies of about 20 different isotopes of hypernuclei have been determined. The accuracy of the energies was between a few ten to a few hundred keV with some systematic ambiguities depending on the emulsion stacks. Hypernuclear events were identified by the unique vertex topologies of their weak decays and analysed by determinations of the lengths and grain densities of the tracks. These results are frequently used to discuss the potential depth of the $\Lambda N$ interaction, the $\Lambda N N$ three-body force due to $\Lambda-\Sigma$ coupling, and the CSB effect in the $\Lambda N$ interaction. A confirmation of the binding energies in light hypernuclear systems is desirable using modern, independent experimental techniques, especially since there is no exact knowledge about the systematic uncertainty for the emulsion data [9].

More recently on the one hand spectrometers with electronic readout systems of the detectors have been employed and on the other hand $\gamma$-ray detectors were used to study excited hypernuclear states. The main experimental techniques to study single $\Lambda$ hypernuclei changed from emulsion to counter experiments. Hypernuclear decay pion measurements with spectrometers were first performed at KEK in the 1980 s. Decay pions from hypernuclei produced by the $\left(K_{\mathrm{stop}}^{-}, \pi^{-}\right)$reaction were observed from several target materials and a ${ }_{\Lambda}^{4} \mathrm{H}$ was identified through its high decay momentum on a continous background of free $\Lambda$ decays and $\Sigma$ decays from quasi-free production [10]. The ${ }_{\Lambda}^{4} \mathrm{H}$ two-body decay peak widths were between 3.0 and $3.8 \mathrm{MeV} / c$ due to the large momentum spread from the energy deposit in the thick targets and the limitation of momentum resolution for the decay-pions. With these widths the absolute momentum could not be determined to better than $100-200 \mathrm{MeV} / c$. A bump in the momentum spectrum was observed that was attributed to the three-body decays of ${ }_{\Lambda}^{4} \mathrm{H},{ }_{\Lambda}^{4} \mathrm{He}$, and ${ }_{\Lambda}^{5} \mathrm{He}$. The hypernuclear fragmentation probabilities for three different targets were reported, yielding important data for discussing the fragmentation process.

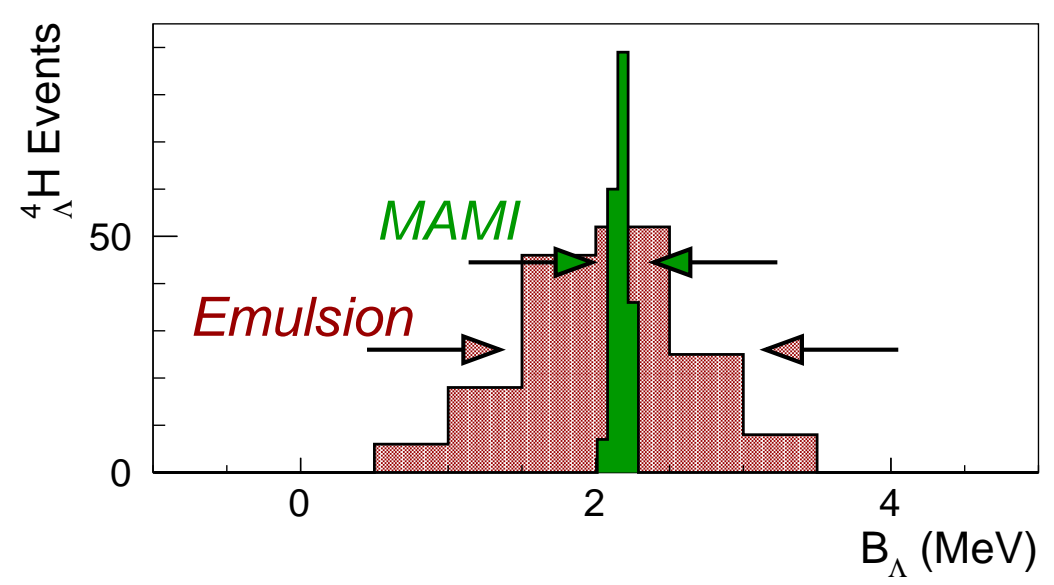

Figure 4: Comparision of the energy resolution for the measurements of the binding energy of ${ }_{\Lambda}^{4} \mathrm{H}$ using the nuclear emulsion technique (data from Ref. [7]) and the high-resolution spectroscopy at MAMI (data from Ref. [12]). An improvement by one order of magnitude was achieved.

The first observation of ${ }_{\Lambda}^{4} \mathrm{H}$ by means of decay-pion spectroscopy with a high resolution magnetic spectrometer was performed 2012 at the Mainz Microtron MAMI [11]. The technique used by the A1 Collaboration was to measure the binding energy of light hypernuclei produced in a multi-step strangeness production, nuclear fragmentation and pionic weak decay reaction chain 
following the $\left(e, e^{\prime} K^{+}\right)$reaction on a ${ }^{9} \mathrm{Be}$ target. In 2014, an extended measurement campaign was performed with improved control over systematic effects and confirming the measurement with two spectrometers at the same time [12]. The resolution of the $\Lambda$ binding energy was designed to be at least better than $100 \mathrm{keV}$ : this is the highest resolution in hypernuclear spectroscopy using magnetic spectrometers. In particular the ${ }_{\Lambda}^{4} \mathrm{H}$ hyperfragment production was considered in the 2014 data taking campaign in which the statistics of the previous 2012 campaign has been significantly increased. Also a re-analysis of the 2012 data has been performed in which the previously published systematic error was decomposed in its stability and calibration contributions. In Fig. 4 the energy resolutions achieved with the two techniques of nuclear emulsion and high-resolution spectroscopy are compared. While the precision has been improved by one order of magnitude, the uncertainty of the high-resolution spectroscopy is currently dominated by systematic errors in the absolute momentum calibration. The main contribution to the total systematic uncertainty was the MAMI beam energy measurement which can be improved further.

\section{Conclusions}

Very recent hypernuclear decay measurements at the electron accelerator MAMI in Germany and at the J-PARC hadron hall in Japan combined with older nuclear emulsion data independently confirmed the existence of strong and spin-dependent $\Lambda$ binding energy differences in the $\left({ }_{\Lambda}^{4} \mathrm{He},{ }_{\Lambda}^{4} \mathrm{H}\right)$ mirror pair of hypernuclei. From the theoretical studies of CSB in $A=4$ hypernuclei it can be concluded that $\Lambda N-\Sigma N$ coupling and three-body forces in the hyperon-nucleon interaction are essential ingredients to the $\Lambda N$ interaction. Hypernuclear physics is a key to understand CSB.

\section{Acknowledgments}

I am indebted to the talented Pepe Gülker for sketching the illustrations of hypernuclei.

\section{References}

[1] A. Bohr, B.R. Mottelson, Nuclear Structure (World Scientific, 1988)

[2] A. Gal, Phys. Lett. B 744, 352 (2015)

[3] S.A. Coon, R.C. Barrett, Phys. Rev. C 36, 2189 (1987)

[4] D. Gazda, A. Gal, Phys. Rev. Lett. 116, 122501 (2016)

[5] R.H. Dalitz, F. von Hippel, Phys. Lett. 10, 153 (1964)

[6] H. Nemura, Y. Akaishi, Y. Suzuki, Phys. Rev. Lett. 89, 142504 (2002)

[7] M. Jurić et al., Nucl. Phys. B 52, 1 (1973)

[8] A. Nogga, Nucl. Phys. A 914, 140 (2013)

[9] D.H. Davis, Nucl. Phys. A 547, 369 (1992)

[10] H. Tamura et al., Phys. Rev. C 40, 479 (1989)

[11] A. Esser et al. (A1 Collaboration), Phys. Rev. Lett. 114, 232501 (2015)

[12] F. Schulz et al. (A1 Collaboration), Nucl. Phys. A (to appear in 2016), doi:10.1016/j.nuclphysa.2016.03.015 